\title{
Solving Bilevel Multiobjective Programming Problem by Elite Quantum Behaved Particle Swarm Optimization
}

\author{
Tao Zhang, ${ }^{1,}{ }^{2}$ Tiesong Hu, ${ }^{1}$ Jia-wei Chen, $^{3}$ \\ Zhongping Wan, ${ }^{3}$ and Xuning Guo ${ }^{1}$ \\ ${ }^{1}$ State Key Laboratory of Water Resource and Hydropower Engineering Science, Wuhan University, \\ Wuhan 430072, China \\ ${ }^{2}$ School of Information and Mathematics, Yangtze University, Jingzhou 434023, China \\ ${ }^{3}$ School of Mathematics and Statistics, Wuhan University, Wuhan 430072, China \\ Correspondence should be addressed to Jia-wei Chen, j.w.chen713@163.com
}

Received 25 August 2012; Accepted 18 October 2012

Academic Editor: Xiaolong Qin

Copyright ( 2012 Tao Zhang et al. This is an open access article distributed under the Creative Commons Attribution License, which permits unrestricted use, distribution, and reproduction in any medium, provided the original work is properly cited.

\begin{abstract}
An elite quantum behaved particle swarm optimization (EQPSO) algorithm is proposed, in which an elite strategy is exerted for the global best particle to prevent premature convergence of the swarm. The EQPSO algorithm is employed for solving bilevel multiobjective programming problem (BLMPP) in this study, which has never been reported in other literatures. Finally, we use eight different test problems to measure and evaluate the proposed algorithm, including low dimension and high dimension BLMPPs, as well as attempt to solve the BLMPPs whose theoretical Pareto optimal front is not known. The experimental results show that the proposed algorithm is a feasible and efficient method for solving BLMPPs.
\end{abstract}

\section{Introduction}

Bilevel programming problem (BLPP) arises in a wide variety of scientific and engineering applications including optimal control, process optimization, game-playing strategy development, transportation problem, and so on. Thus the BLPP has been developed and researched by many scholars. The reviews, monographs, and surveys on the BLPP can refer to [1-11]. Moreover, the evolutionary algorithms (EAs) have been employed to address BLPP in papers [12-16].

For the multiobjective characteristics widely existing in the BLPP, the bilevel multiobjective programming problem (BLMPP) has attracted many researchers to study 
it. For example, Shi and Xia [17, 18], Abo-Sinna and Baky [19], Nishizaki and Sakawa [20], Zheng et al. [21] presented an interactive algorithm for BLMPP. Eichfelder [22] presented a method for solving nonlinear bilevel multiobjective optimization problems with coupled upper level constraints. Thereafter, Eichfelder [23] developed a numerical method for solving nonlinear nonconvex bilevel multiobjective optimization problems. In recent years, the metaheuristic has attracted considerable attention as an alternative method for BLMPP. For example, Deb and Sinha [24-26], as well as Sinha and Deb [27] discussed BLMPP based on evolutionary multiobjective optimization principles. Based on those studies, Deb and Sinha [28] proposed a viable and hybrid evolutionarylocal-search based algorithm and presented challenging test problems. Sinha [29] presented a progressively interactive evolutionary multiobjective optimization method for BLMPP.

Particle swarm optimization (PSO) is a relatively novel heuristic algorithm inspired by the choreography of a bird flock, which has been found to be quite successful in a wide variety of optimization tasks [30]. Due to its high speed of convergence and relative simplicity, the PSO algorithm has been employed by many researchers for solving BLPPs. For example, Li et al. [31] proposed a hierarchical PSO for solving BLPP. Kuo and Huang [32] applied the PSO algorithm for solving bilevel linear programming problem. Gao et al. [33] presented a method to solve bilevel pricing problems in supply chains using PSO. However, it is worth noting that the papers mentioned above only for bilevel single objective problems and the BLMPP have seldom been studied using PSO so far. There are probably two reasons for this situation. One reason is that the added complexities associated with solving each level, and the other reason is that the global convergence of the PSO cannot be guaranteed [34].

In this paper, a global convergence guaranteed method called as EQPSO is proposed, in which an elite strategy is exerted for global best particle to prevent premature convergence of the swarm. The EQPSO is employed for solving the BLMPP in this study, which has not been reported in other literatures. For such problems, the proposed algorithm directly simulates the decision process of the bilevel programming, which is different from most traditional algorithms designed for specific versions or based on specific assumptions. The BLMPP is transformed to solve multiobjective optimization problems in the upper level and the lower level interactively by the EQPSO. And a set of approximate Pareto optimal solutions for BLMPP are obtained using the elite strategy. This interactive procedure is repeated until the accurate Pareto optimal solutions of the original problem are found. The rest of the paper is organized as follows. In Section 2, the problem formulation is provided. The proposed algorithm for solving bilevel multiobjective problem is presented in Section 3. In Section 4, some numerical examples are given to demonstrate the feasibility and efficiency of the proposed algorithm.

\section{Problem Formulation}

Let $x \in R^{n_{1}}, y \in R^{n_{2}}, F: R^{n_{1}} \times R^{n_{2}} \rightarrow R^{m_{1}}, f: R^{n_{1}} \times R^{n_{2}} \rightarrow R^{m_{2}}, G: R^{n_{1}} \times R^{n_{2}} \rightarrow R^{p}$, $g: R^{n_{1}} \times R^{n_{2}} \rightarrow R^{q}$. The general model of the BLMPP can be written as follows:

$$
\begin{aligned}
& \min _{x} F(x, y) \\
& \text { s.t. } \quad G(x, y) \geq 0,
\end{aligned}
$$




$$
\begin{array}{ll}
\min _{y} & f(x, y) \\
\text { s.t. } & g(x, y) \geq 0,
\end{array}
$$

where $F(x, y)$ and $f(x, y)$ are the upper level and the lower level objective functions, respectively. $G(x, y)$ and $g(x, y)$ denote the upper level and the lower level constraints, respectively.

Let $S=\{(x, y) \mid G(x, y) \geq 0, g(x, y) \geq 0\}, X=\{x \mid \exists y, G(x, y) \geq 0, g(x, y) \geq 0\}$, $S(x)=\{y \mid g(x, y) \geq 0\}$, and for the fixed $x \in X$, let $\bar{S}(X)$ denote the weak efficiency set of solutions to the lower level problem, the feasible solution set of problem (2.1) is denoted as: $\operatorname{IR}=\{(x, y) \mid(x, y) \in S, y \in \bar{S}(X)\}$.

Definition 2.1. For a fixed $x \in X$, if $y$ is a Pareto optimal solution to the lower level problem, then $(x, y)$ is a feasible solution to the problem (2.1).

Definition 2.2. If $\left(x^{*}, y^{*}\right)$ is a feasible solution to the problem (2.1), and there are no $(x, y) \in \operatorname{IR}$, such that $F(x, y) \prec F\left(x^{*}, y^{*}\right)$, then $\left(x^{*}, y^{*}\right)$ is a Pareto optimal solution to the problem (2.1), where " $<$ " denotes Pareto preference.

For problem (2.1), it is noted that a solution $\left(x^{*}, y^{*}\right)$ is feasible for the upper level problem if and only if $y^{*}$ is an optimal solution for the lower level problem with $x=x^{*}$. In practice, we often make the approximate Pareto optimal solutions of the lower level problem as the optimal response feed back to the upper level problem, and this point of view is accepted usually. Based on this fact, the EQPSO algorithm may have a great potential for solving BLMPP. On the other hand, unlike the traditional point-by-point approach mentioned in Section 1, the EQPSO algorithm uses a group of points in its operation, thus the EQPSO can be developed as a new way for solving BLMPP. We next present the algorithm based on the EQPSO is presented for (2.1).

\section{The Algorithm}

\subsection{The EQPSO}

The quantum behaved particle swarm optimization (QPSO) is the integration of PSO and quantum computing theory developed by [35-38]. Compared with PSO, it needs no velocity vectors for particles and has fewer parameters to adjust. Moreover, its global convergence can be guaranteed [39]. Due to its global convergence and relative simplicity, it has been found to be quite successful in a wide variety of optimization tasks. For example, a wide range of continuous optimization problems [40-45] are solved by QPSO and the experiment results show that the QPSO works better than standard PSO. Some improved QPSO algorithms can refer to [46-48]. In this paper, the EQPSO algorithm is proposed, in which an elite strategy is exerted for global best particle to prevent premature convergence of the swarm, and it makes the proposed algorithm has good performance for solving the high dimension BLMPPS. The EQPSO has the same design principle with the QPSO except for the global optimal particle selection criterion, so the global convergence proof of the 
EQPSO can refer to [39]. In the EQPSO, the particles move according to the following iterative equation:

$$
\begin{aligned}
& z^{t+1}=p^{t}-\alpha^{t}\left(m \text { Best }^{t}-z^{t}\right) * l_{n}\left(\frac{1}{u}\right) \quad \text { if } k \geq 0.5, \\
& z^{t+1}=p^{t}+\alpha^{t}\left(m \text { Best }^{t}-z^{t}\right) * l_{n}\left(\frac{1}{u}\right) \quad \text { if } k<0.5,
\end{aligned}
$$

where

$$
\begin{gathered}
p^{t}=\varphi * p_{p \text { Best }}^{t}+(1-\varphi) * p_{g \text { Best }}^{t} \\
m \text { Best }^{t}=\frac{1}{N} \sum_{i=1}^{N} p_{p \text { Best }_{i^{\prime}}}{ }^{\prime} \\
\alpha^{t}=m-(m-n) * \frac{t}{T} \\
p_{g \text { Best }} \in \operatorname{rand}\left(A_{t}\right),
\end{gathered}
$$

where the $z$ denotes the particle's position. $m$ Best denotes the mean best position of all the particles' best positions. The $k, u$, and $\varphi$ are random numbers distributed uniformly on $(0,1)$, respectively. $\alpha(t)$ is the expansion-contraction coefficient. In general, $m=1, n=$ $0.5, t$ is the current iteration number, and $T$ is the maximum number of iterations. The $p_{p \text { Best }}$ and $p_{g \text { Best }}$ are the particle's personal best position and the global best position, respectively. $A_{t}$ is the elite set which is introduced in following parts (see Algorithm: Step 3).

\subsection{The Algorithm for Solving BLMPP}

The process of the proposed algorithm for solving BLMPP is an interactive coevolutionary process. We first initialize population and then solve multiobjective optimization problems in the upper level and the lower level interactively using the EQPSO. For one time of iteration, a set of approximate Pareto optimal solutions for problem 1 is obtained by the elite strategy which was adopted in Deb et al. [49]. This interactive procedure is repeated until the accurate Pareto optimal solutions of problem (2.1) are found. The details of the proposed algorithm are given as follows.

\section{Algorithm}

Step 1. Initializing.

Step 1.1. Initialize the population $P_{0}$ with $N_{u}$ particles which is composed by $n_{s}=N_{u} / N_{l}$ subswarms of size $N_{l}$ each. The particle's position of the $k$ th $\left(k=1,2, \ldots, n_{s}\right)$ subswarm is presented as: $z_{j}=\left(x_{j}, y_{j}\right)\left(j=1,2, \ldots, n_{l}\right)$ and $z_{j}$ is sampled randomly in the feasible space.

Step 1.2. Initialize the external loop counter $t:=0$. 
Step 2. For the $k$ th subswarm, $\left(k=1,2, \ldots, n_{s}\right)$, each particle is assigned a nondomination rank $\mathrm{ND}_{l}$ and a crowding value $\mathrm{CD}_{l}$ in $f$ space. Then, all resulting subswarms are combined into one population which is named as the $P_{t}$. Afterwards, each particle is assigned a nondomination rank $\mathrm{ND}_{u}$ and a crowding value $\mathrm{CD}_{u}$ in $F$ space.

Step 3. The nondomination particles assigned both $\mathrm{ND}_{u}=1$ and $\mathrm{ND}_{l}=1$ from $P_{t}$ are saved in the elite set $A_{t}$.

Step 4 . For the $k$ th subswarm, $\left(k=1,2, \ldots, n_{s}\right)$, update the lower level decision variables.

Step 4.1. Initialize the lower level loop counter $t_{l}:=0$.

Step 4.2. Update the $j$ th $\left(j=1,2, \ldots, N_{l}\right)$ particle's position with the fixed $x_{j}$ according to (3.1) and (3.2).

Step 4.3. $t_{l}:=t_{l}+1$.

Step 4.4. If $t_{l} \geq T_{l}$, go to Step 4.5. Otherwise, go to Step 4.2

Step 4.5. Each particle of the $i$ th subswarm is reassigned a nondomination rank $\mathrm{ND}_{l}$ and a crowding value $\mathrm{CD}_{l}$ in $f$ space. Then, all resulting subswarms are combined into one population which is renamed as the $Q_{t}$. Afterwards, each particle is reassigned a nondomination rank $\mathrm{ND}_{u}$ and a crowding value $\mathrm{CD}_{u}$ in $F$ space.

Step 5. Combined population $P_{t}$ and $Q_{t}$ to form $R_{t}$. The combined population $R_{t}$ is reassigned a nondomination rank $\mathrm{ND}_{u}$, and the particles within an identical nondomination rank are assigned a crowding distance value $\mathrm{CD}_{u}$ in the $F$ space.

Step 6. Choose half particles from $R_{t}$. The particles of rank $\mathrm{ND}_{u}=1$ are considered first. From the particles of rank $\mathrm{ND}_{u}=1$, the particles with $\mathrm{ND}_{l}=1$ are noted one by one in the order of reducing crowding distance $\mathrm{CD}_{u}$, for each such particle the corresponding subswarm from its source population (either $P_{t}$ or $Q_{t}$ ) is copied in an intermediate population $S_{t}$. If a subswarm is already copied in $S_{t}$ and a future particle from the same subswarm is found to have $\mathrm{ND}_{u}=\mathrm{ND}_{l}=1$, the subswarm is not copied again. When all particles of $\mathrm{ND}_{u}=1$ are considered, a similar consideration is continued with $\mathrm{ND}_{u}=2$ and so on till exactly $n_{s}$ subswarms are copied in $S_{t}$.

Step 7. Update the elite set $A_{t}$. The nondomination particles assigned both $\mathrm{ND}_{u}=1$ and $\mathrm{ND}_{l}=1$ from $S_{t}$ are saved in the elite set $A_{t}$.

Step 8. Update the upper level decision variables in $S_{t}$.

Step 8.1. Initiate the upper level loop counter $t_{u}:=0$.

Step 8.2. Update the $i$ th $\left(i=1,2, \ldots, N_{u}\right)$ particle's position with the fixed $y_{i}$ according to (3.1) and (3.2).

Step 8.3. $t_{u}:=t_{u}+1$.

Step 8.4. If $t_{u} \geq T_{u}$, go to Step 8.5. Otherwise, go to Step 8.2.

Step 8.5. Every member is then assigned a nondomination rank $\mathrm{ND}_{u}$ and a crowding distance value $\mathrm{CD}_{u}$ in $F$ space. 
Step 9. $t:=t+1$.

Step 10. If $t \geq T$, output the elite set $A_{t}$. Otherwise, go to Step 2 .

In Steps 4 and 8 , the global best position is chosen at random from the elite set $A_{t}$. The criterion of personal best position choice is that, if the current position is dominated by the previous position, then the previous position is kept; otherwise, the current position replaces the previous one; if neither of them is dominated by the other, then we select one of them randomly. A relatively simple scheme is used to handle constraints. Whenever two individuals are compared, their constraints are checked. If both are feasible, nondomination sorting technology is directly applied to decide which one is selected. If one is feasible and the other is infeasible, the feasible dominates. If both are infeasible, then the one with the lowest amount of constraint violation dominates the other. Notations used in the proposed algorithm are detailed in Table 1.

\section{Numerical Experiment}

In this section, three examples will be considered to illustrate the feasibility of the proposed algorithm for problem (2.1). In order to evaluate the closeness between the obtained Pareto optimal front and the theoretical Pareto optimal front, as well as the diversity of the obtained Pareto optimal solutions along the theoretical Pareto optimal front, we adopted the following evaluation metrics.

\subsection{Performance Evaluation Metrics}

(a) Generational Distance (GD): this metric used by Deb [50] is employed in this paper as a way of evaluating the closeness between the obtained Pareto optimal front and the theoretical Pareto optimal front. The GD metric denotes the average distance between the obtained Pareto optimal front and the theoretical Pareto optimal front:

$$
\mathrm{GD}=\frac{\sqrt{\sum_{i=1}^{n} d_{i}^{2}}}{n}
$$

where $n$ is the number of the obtained Pareto optimal solutions by the proposed algorithm and $d_{i}$ is the Euclidean distance between each obtained Pareto optimal solution and the nearest member of the theoretical Pareto optimal set.

(b) Spacing (SP): this metric is used to evaluate the diversity of the obtained Pareto optimal solutions by comparing the uniform distribution and the deviation of solutions as described by Deb [50]:

$$
\mathrm{SP}=\frac{\sum_{m=1}^{M} d_{m}^{e}+\sum_{i=1}^{n}\left(\bar{d}-d_{i}\right)^{2}}{\sum_{m=1}^{M} d_{m}^{e}+n \bar{d}}
$$

where $d_{i}=\min _{j}\left(\left|F_{1}^{i}(x, y)-F_{1}^{j}(x, y)\right|+\left|F_{2}^{i}(x, y)-F_{2}^{j}(x, y)\right|\right), i, j=1,2, \ldots, n, \bar{d}$ is the mean of all $d_{i}, d_{m}^{e}$ is the Euclidean distance between the extreme solutions in obtained Pareto optimal solution set and the theoretical Pareto optimal solution set on the $m$ th objective, $M$ is the 
Table 1: The notations of the algorithm.

\begin{tabular}{ll}
\hline$x_{i}$ & The $i$ th particle's position of the upper level problem. \\
$y_{j}$ & The $j$ th particle's position of the lower level problem. \\
$z_{j}$ & The $j$ th particle's position of BLMPP. \\
$N_{u}$ & The population size of the upper level problem. \\
$N_{l}$ & The sub-swarm size of the lower level problem. \\
$t$ & Current iteration number for the overall problem. \\
$T$ & The predefined max iteration number for $t$. \\
$t_{u}$ & Current iteration number for the upper level problem. \\
$t_{l}$ & Current iteration number for the lower level problem. \\
$T_{u}$ & The predefined max iteration number for $t_{u}$. \\
$T_{l}$ & The predefined max iteration number for $t_{l}$. \\
$\mathrm{ND}_{u}$ & Non-domination sorting rank of the upper level problem. \\
$\mathrm{CD}_{u}$ & Crowding distance value of the upper level problem. \\
$\mathrm{ND}_{l}$ & Non-domination sorting rank of the lower level problem. \\
$\mathrm{CD}_{l}$ & Crowding distance value of the lower level problem. \\
$P_{t}$ & The th iteration population. \\
$Q_{t}$ & The offspring of $P_{t}$. \\
$S_{t}$ & Intermediate population. \\
\hline
\end{tabular}

number of the upper level objective function, and $n$ is the number of the obtained solutions by the proposed algorithm.

All results presented in this paper have been obtained on a personal computer (CPU: AMD Phenon II X6 1055T 2.80 GHz; RAM: 3.25GB) using a c\# implementation of the proposed algorithm.

\subsection{Numerical Examples}

\subsubsection{Low Dimension BLMPPS}

Example 4.1. Example 4.1 is taken from [22]. Here $x \in R^{1}, y \in R^{2}$. In this example, the population size and iteration times are set as follows: $N_{u}=200, T_{u}=200, N_{l}=40, T_{l}=40$, and $T=40$ :

$$
\begin{array}{ll}
\min _{x} & F(x, y)=\left(y_{1}-x, y_{2}\right) \\
\text { s.t. } & G_{1}(y)=1+y_{1}+y_{2} \geq 0 \\
\min _{y} & f(x, y)=\left(y_{1}, y_{2}\right) \\
\text { s.t. } & g_{1}(x, y)=x^{2}-y_{1}^{2}-y_{2}^{2} \geq 0, \\
& -1 \leq y_{1}, \quad y_{2} \leq 1, \quad 0 \leq x \leq 1 .
\end{array}
$$

Figure 1 shows the obtained Pareto front of this example by the proposed algorithm. From Figure 1, it can be seen that the obtained Pareto front is very close to the theoretical 


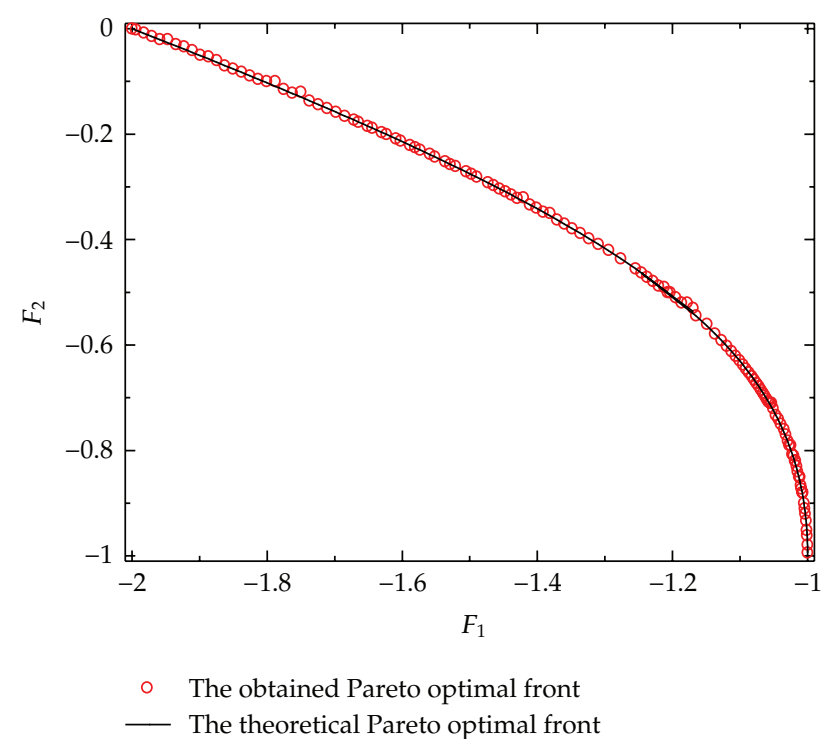

Figure 1: The obtained Pareto optimal front of Example 4.1.

Pareto optimal front, and the average distance between the obtained Pareto optimal front and the theoretical Pareto optimal front is 0.00024 , that is, GD $=0.00024$ (see Table 2). Moreover, the lower SP value ( $\mathrm{SP}=0.0042$, see Table 2 ) shows that the proposed algorithm is able to obtain a good distribution of solutions on the entire range of the theoretical Pareto optimal front. Figure 2 shows the obtained solutions of this example, which follow the relationship, that is, $y_{1}=-1-y_{2}, y_{2}=-1 / 2 \pm(1 / 4) \sqrt{8 x^{2}-4}$ and $x \in(1 / \sqrt{2}, 1)$. It is also obvious that all obtained solutions are close to being on the upper level constraint $G(x)$ boundary $\left(1+y_{1}+y_{2}=\right.$ $0)$.

Example 4.2. Example 4.2 is taken from [51]. Here $x \in R^{1}, y \in R^{2}$. In this example, the population size and iteration times are set as follows: $N_{u}=200, T_{u}=50, N_{l}=40, T_{l}=20$, and $T=40$ :

$$
\begin{gathered}
\min _{x} F(x, y)=\left(x^{2}+\left(y_{1}-1\right)^{2}+y_{2}^{2},(x-1)^{2}+\left(y_{1}-1\right)^{2}+y_{2}^{2}\right) \\
\min _{y} f(x, y)=\left(y_{1}^{2}+y_{2}^{2},\left(y_{1}-x\right)^{2}+y_{2}^{2}\right) \\
-1 \leq x, \quad y_{1}, y_{2} \leq 2 .
\end{gathered}
$$

Figure 3 shows the obtained Pareto optimal front of this example by the proposed algorithm. From Figure 3, it is obvious that the obtained Pareto optimal front is very close to the theoretical Pareto optimal front, the average distance between the obtained Pareto optimal front and the theoretical Pareto optimal front is 0.00003 (see Table 2). On the other hand, the obtained Pareto optimal solutions can distribute uniformly on entire range of theoretical Pareto optimal front base on the fact that the SP value is lower (SP $=0.00169$, see Table 2). 


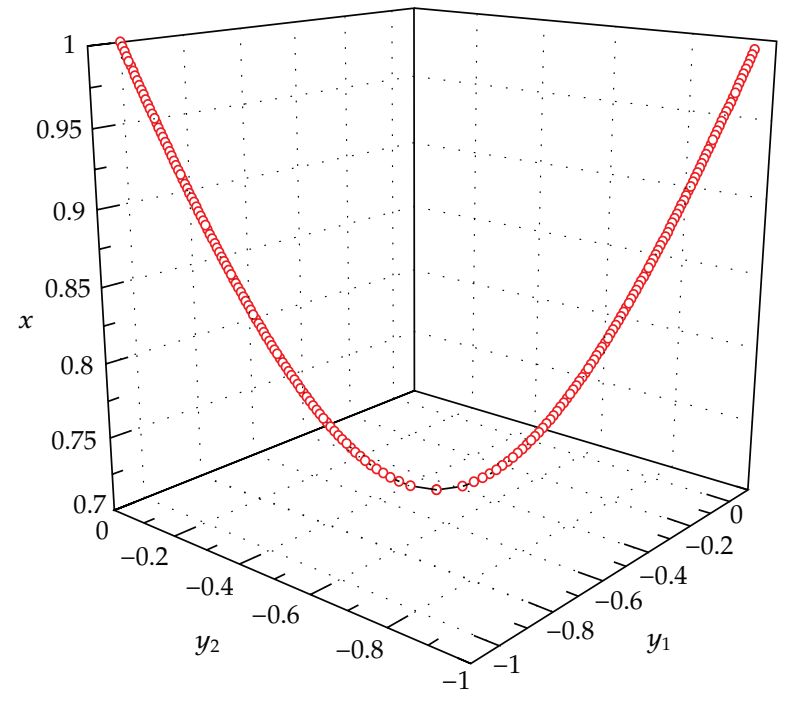

- The obtained Pareto optimal solutions

- The theoretical Pareto optimal solutions

Figure 2: The obtained solutions of Example 4.1.

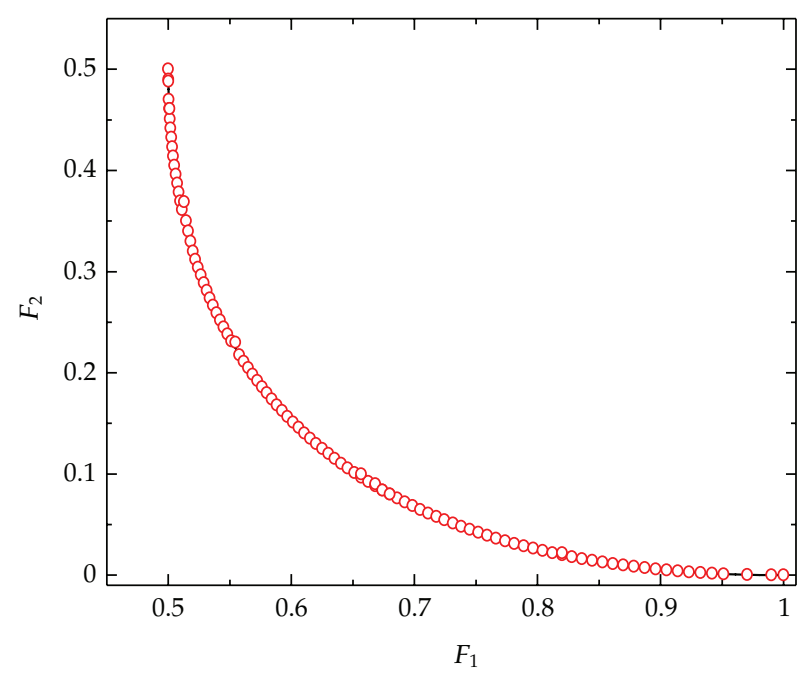

- The obtained Pareto optimal front

— The theoretical Pareto optimal front

Figure 3: The obtained Pareto optimal front of Example 4.2.

Figure 4 shows the obtained Pareto optimal solutions, they follow the relationship, that is, $x=y_{1}, y_{1} \in[0.5,1]$ and $y_{2}=0$. 


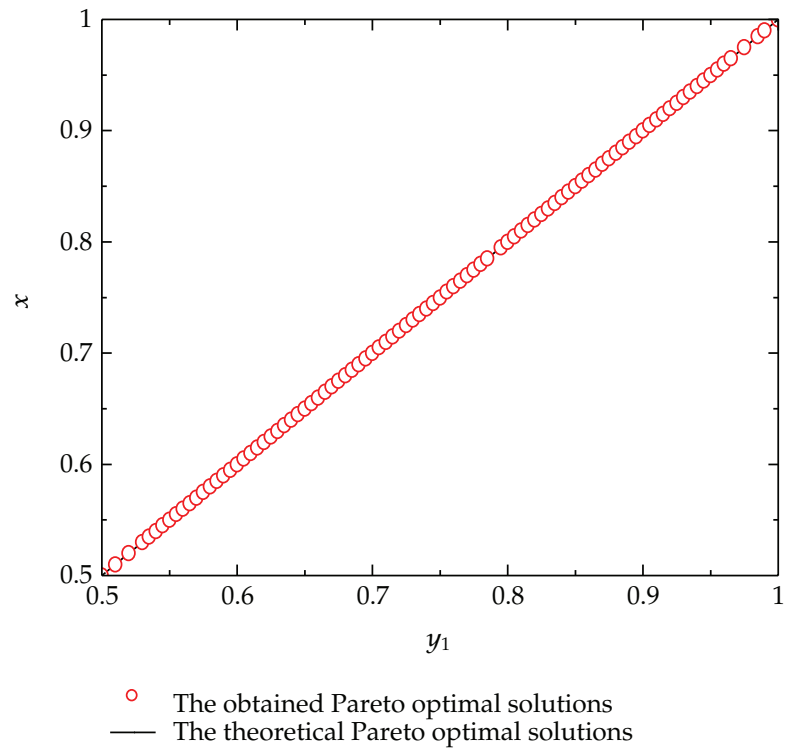

Figure 4: The obtained solutions Example 4.2.

\subsubsection{High Dimension BLMPPS}

Example 4.3. Example 4.3 is taken from [28]. Here $x \in R^{10}, y \in R^{10}$. In this example, the population size and iteration times are set as follows: $N_{u}=400, T_{u}=50, N_{l}=40, T_{l}=20$, and $T=60$ :

$$
\begin{aligned}
\min _{x} F(x, y)= & \left(1+r-\cos \left(\alpha \pi x_{1}\right)\right)+\sum_{j=2}^{K}\left(x_{j}-\frac{j-1}{2}\right)^{2}+\tau \sum_{i=2}^{K}\left(y_{i}-x_{i}\right)^{2}-r \cos \left(r \frac{\pi x_{1}}{2 y_{1}}\right), \\
& \left(1+r-\sin \left(\alpha \pi x_{1}\right)\right)+\sum_{j=2}^{K}\left(x_{j}-\frac{j-1}{2}\right)^{2} \\
& \left.+\tau \sum_{i=2}^{K}\left(y_{i}-x_{i}\right)^{2}-r \sin \left(r \frac{\pi x_{1}}{2 y_{1}}\right)\right) \\
\min _{y} f(x, y)= & \left(y_{1}^{2}+\sum_{i=2}^{K}\left(y_{i}-x_{i}\right)^{2}+\sum_{i=2}^{K} 10\left(1-\cos \left(\frac{\pi}{k}\left(y_{i}-x_{i}\right)\right)\right)\right. \\
& \left.\sum_{i=1}^{K}\left(y_{i}-x_{i}\right)^{2}+\sum_{i=2}^{K} 10\left|1-\sin \left(\frac{\pi}{k}\left(y_{i}-x_{i}\right)\right)\right|\right)
\end{aligned}
$$

s.t. $\quad-K \leq y_{i} \leq K, \quad(i=1,2, \ldots, K)$;

$1 \leq x_{1} \leq 4, \quad-K \leq x_{j} \leq K, \quad(j=2,3, \ldots, K)$.

$\alpha=1, \quad r=0.1, \quad \tau=1, \quad \gamma=1, \quad K=10$. 
Example 4.4. Example 4.4 is taken from [28]. Here $x \in R^{10}, y \in R^{10}$. In this example, the population size and iteration times are set as follows: $N_{u}=400, T_{u}=50, N_{l}=40, T_{l}=20$, and $T=80$.

$$
\begin{gathered}
\min _{x} F(x, y)=v_{1}\left(x_{1}\right)+\sum_{j=2}^{K} y_{j}^{2}+10\left(1-\cos \left(\frac{\pi}{k}\right) y_{i}\right)+\tau \sum_{i=2}^{K}\left(y_{i}-x_{i}\right)^{2}-r \cos \left(r \frac{\pi x_{1}}{2 y_{1}}\right), \\
v_{2}\left(x_{1}\right)+\sum_{j=2}^{K} y_{j}^{2}+10\left(1-\cos \left(\frac{\pi}{k}\right) y_{i}\right) \\
+\tau \sum_{i=2}^{K}\left(y_{i}-x_{i}\right)^{2}-r \sin \left(r \frac{\pi x_{1}}{2 y_{1}}\right), \\
\min _{y} \quad f(x, y)=\left(y_{1}^{2}+\sum_{i=2}^{K}\left(y_{i}-x_{i}\right)^{2}, \sum_{i=1}^{K} i\left(y_{i}-x_{i}\right)^{2}\right) \\
\text { s.t. } \quad-K \leq y_{i} \leq K, \quad(i=1,2, \ldots, K) ; \\
\quad 0.001 \leq x_{1} \leq 4, \quad-K \leq x_{j} \leq K, \quad(j=2,3, \ldots, K) .
\end{gathered}
$$

where

$$
\begin{aligned}
& v_{1}\left(x_{1}\right)= \begin{cases}\cos (0.2 \pi) x_{1}+\sin (0.2 \pi) \sqrt{\left|0.02 \sin \left(5 \pi x_{1}\right)\right|}, & \text { for } 0 \leq x_{1} \leq 1, \\
x_{1}-(1-\sin (0.2 \pi)), & \text { for } x_{1}>1,\end{cases} \\
& v_{2}\left(x_{1}\right)= \begin{cases}-\sin (0.2 \pi) x_{1}+\cos (0.2 \pi) \sqrt{\left|0.02 \sin \left(5 \pi x_{1}\right)\right|}, & \text { for } 0 \leq x_{1} \leq 1, \\
0.1\left(x_{1}-1\right)-\sin (0.2 \pi), & \text { for } x_{1}>1 .\end{cases}
\end{aligned}
$$

This problem is more difficult compared to the previous problems (Examples 4.1 and 4.2 ) because the lower level problem of this example has multimodalities, thereby making the lower level problem difficult in finding the upper level Pareto optimal front. From Figure 5, it can be seen that the obtained Pareto front is very close to the theoretical Pareto optimal front, and the average distance between the obtained Pareto optimal front and the theoretical Pareto optimal front is 0.00027, that is, GD $=0.00027$ (see Table 2). Moreover, the lower SP value (SP $=0.00127$, see Table 2 ) shows that the proposed algorithm is able to obtain a good distribution of solutions on the entire range of the theoretical Pareto optimal front. Furthermore, two obtained lower level Pareto optimal fronts are given when $x_{1}=2$ and $x_{1}=2.5$.

Figure 6 shows the obtained Pareto front of Example 4.4 by the proposed algorithm. The upper level problem has multimodalities, thereby causing an algorithm difficulty in finding the upper level Pareto optimal front. From Figure 6, it can be seen that the obtained Pareto front is very close to the theoretical Pareto optimal front, and the average distance between the obtained Pareto optimal front and the theoretical Pareto optimal front is 0.00036 , that is, GD $=0.00036$ (see Table 2). Moreover, the lower SP value $(\mathrm{SP}=0.00235$, see Table 2) 


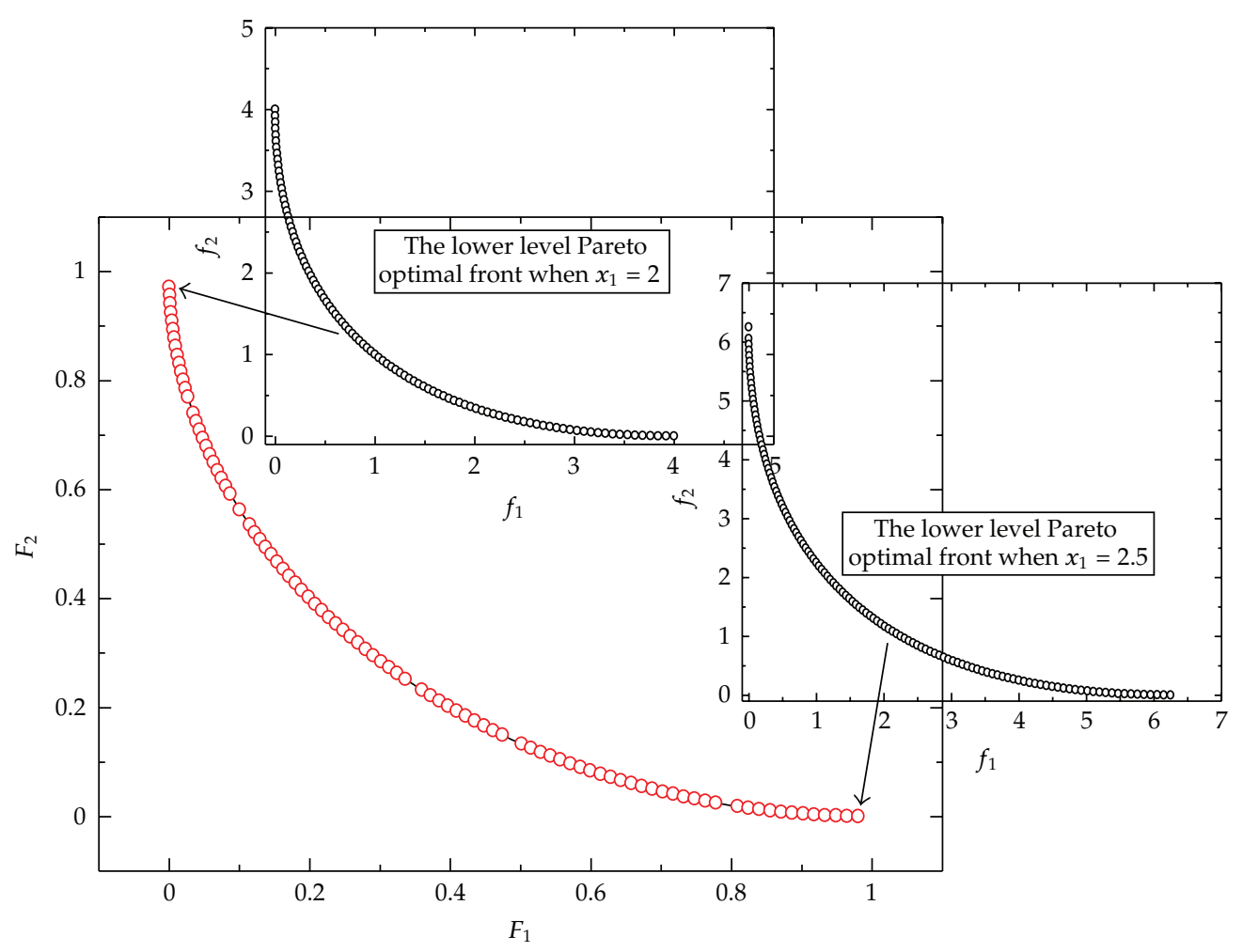

O The obtained Pareto optimal solutions

— The theoretical Pareto optimal solutions

Figure 5: The obtained Pareto front of Example 4.3.

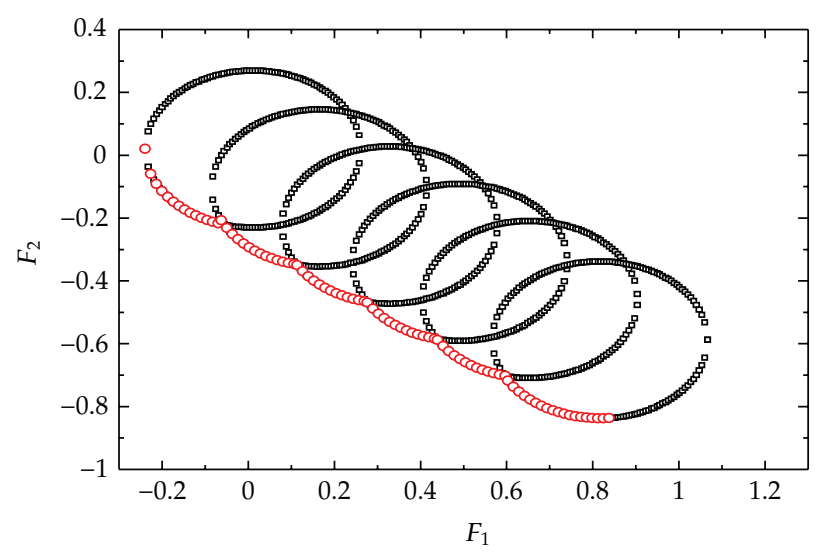

$\square$ The theoretical Pareto optimal front of lower level problem

- The obtained upper level Pareto optimal front

Figure 6: The obtained Pareto front of Example 4.4. 
Table 2: Results of the Generation Distance (GD) and Spacing (SP) metrics for the above six examples.

\begin{tabular}{lcr}
\hline Example & GD & SP \\
\hline Example 4.1 & 0.00024 & 0.00442 \\
Example 4.2 & 0.00003 & 0.00169 \\
Example 4.3 & 0.00027 & 0.00127 \\
Example 4.4 & 0.00036 & 0.00235 \\
Example 4.5 & 0.00058 & 0.00364 \\
Example 4.6 & 0.00039 & 0.00168 \\
\hline
\end{tabular}

shows that the proposed algorithm is able to obtain a good distribution of solutions on the entire range of the theoretical Pareto optimal front. Furthermore, all corresponding lower level Pareto optimal fronts are given.

Example 4.5. Example 4.5 is taken from [28]. Here $x \in R^{10}, y \in R^{10}$. In this example, the population size and iteration times are set as follows: $N_{u}=400, T_{u}=50, N_{l}=40, T_{l}=20$, and $T=60$ :

$$
\begin{array}{r}
\min _{x} F(x \cdot y)=\left(x_{1}+\sum_{j=3}^{K}\left(x_{j}-\frac{j}{2}\right)^{2}+\tau \sum_{i=3}^{K}\left(y_{i}-x_{i}\right)^{2}-\cos \left(4 \tan ^{-1}\left(\frac{x_{2}-y_{2}}{x_{1}-y_{1}}\right)\right),\right. \\
\left.x_{2}+\sum_{j=3}^{K}\left(x_{j}-\frac{j}{2}\right)^{2}+\tau \sum_{i=3}^{K}\left(y_{i}-x_{i}\right)^{2}-\cos \left(4 \tan ^{-1}\left(\frac{x_{2}-y_{2}}{x_{1}-y_{1}}\right)\right)\right)
\end{array}
$$

s.t. $G(x)=x_{2}-\left(1-x_{1}^{2}\right)^{2} \geq 0$

$$
\min _{y} f(x, y)=\left(y_{1}+\sum_{i=3}^{K}\left(y_{i}-x_{i}\right)^{2}, y_{2}+\sum_{i=3}^{K}\left(y_{i}-x_{i}\right)^{2}\right)
$$

s.t. $\quad g_{1}(x, y)=\left(y_{1}-x_{1}\right)^{2}+\left(y_{2}-x_{2}\right)^{2} \leq r^{2}$

$$
\begin{aligned}
& R\left(x_{1}\right)=\left(0.1+0.15\left|\sin \left(2 \pi\left(x_{1}-0.1\right)\right)\right|\right), \\
& -K \leq y_{i} \leq K, \quad(i=1,2, \ldots, K) ; \\
& 0 \leq x_{1} \leq K, \quad-K \leq x_{j} \leq K, \quad(j=2,3, \ldots, K) . \\
& \tau=1, \quad r=0.2, \quad K=10 .
\end{aligned}
$$

Example 4.6. Example 4.6 is taken from [28]. Here $x \in R^{1}, y \in R^{9}$. In this example, the population size and iteration times are set as follows: $N_{u}=400, T_{u}=50, N_{l}=40, T_{l}=20$, and $T=40$ :

$$
\begin{array}{ll}
\min _{x} & F(x, y)=\left(\left(1-y_{1}\right)\left(1+\sum_{j=2}^{K} y_{j}^{2}\right) x_{1}, y_{1}\left(1+\sum_{j=2}^{K} y_{j}^{2}\right) x_{1}\right) \\
\text { s.t. } & G_{1}(x, y)=-\left(1-y_{1}\right) x_{1}-\frac{1}{2} x_{1} y_{1} \leq 1
\end{array}
$$




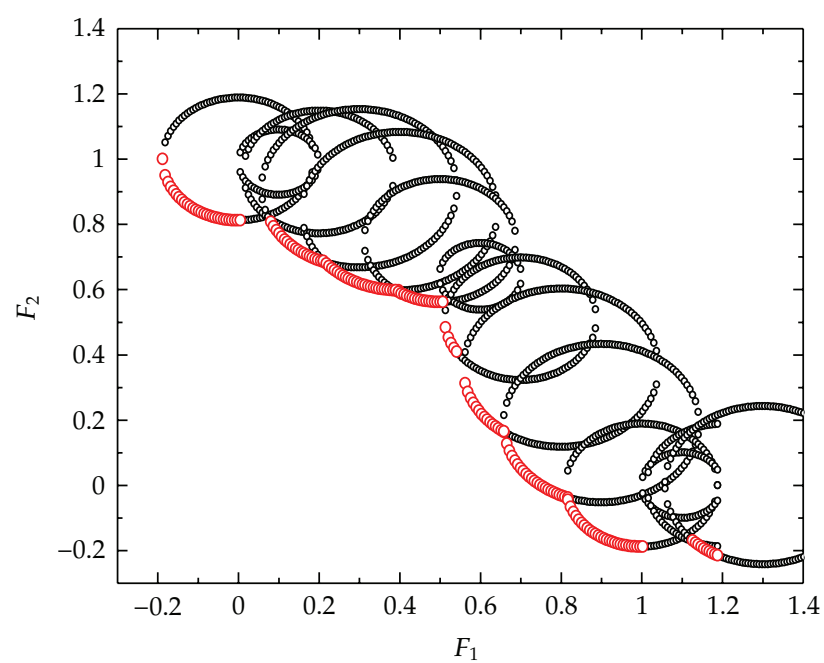

- The theoretical Pareto optimal front of lower level problem

The obtained upper level Pareto optimal front

Figure 7: The obtained Pareto front of Example 4.5.

$$
\begin{array}{ll}
\min _{y} \quad f(x, y)=\left(\left(1-y_{1}\right)\left(1+\sum_{j=K+1}^{K+L} y_{j}^{2}\right) x_{1}, y_{1}\left(1+\sum_{j=K+1}^{K+L} y_{j}^{2}\right) x_{1}\right) \\
\text { s.t. } \quad 1 \leq x_{1} \leq 2, \quad-1 \leq y_{1} \leq 1, \\
\quad-(K+L) \leq y_{j} \leq(K+L), \quad(j=2,3, \ldots, K+L), \quad K=5, \quad L=4 .
\end{array}
$$

Figure 7 shows the obtained Pareto front of Example 4.5 by the proposed algorithm. From Figure 7, it can be seen that the obtained Pareto front is very close to the theoretical Pareto optimal front, and the average distance between the obtained Pareto optimal front and the theoretical Pareto optimal front is 0.00058 , that is, GD $=0.00058$ (see Table 2). Moreover, the lower SP value (SP $=0.00364$, see Table 2 ) shows that the proposed algorithm is able to obtain a good distribution of solutions on the entire range of the theoretical Pareto optimal front. Furthermore, all obtained lower level Pareto optimal fronts are given. It is also obvious that the Pareto optimal fronts for both the lower and upper level lie on constraint boundaries and every lower level Pareto optimal front has an unequal contribution to the upper level Pareto optimal front.

Figure 8 shows the obtained Pareto front of Example 4.6 by the proposed algorithm. From Figure 8, it can be seen that the obtained Pareto front is very close to the theoretical Pareto optimal front, and the average distance between the obtained Pareto optimal front and the theoretical Pareto optimal front is 0.00039 , that is, GD $=0.00039$ (see Table 2). Moreover, the lower SP value (SP $=0.00168$, see Table 2 ) shows that the proposed algorithm is able to obtain a good distribution of solutions on the entire range of the theoretical Pareto optimal front. Furthermore, three obtained lower level Pareto optimal fronts are given when $y_{1}=1$, 


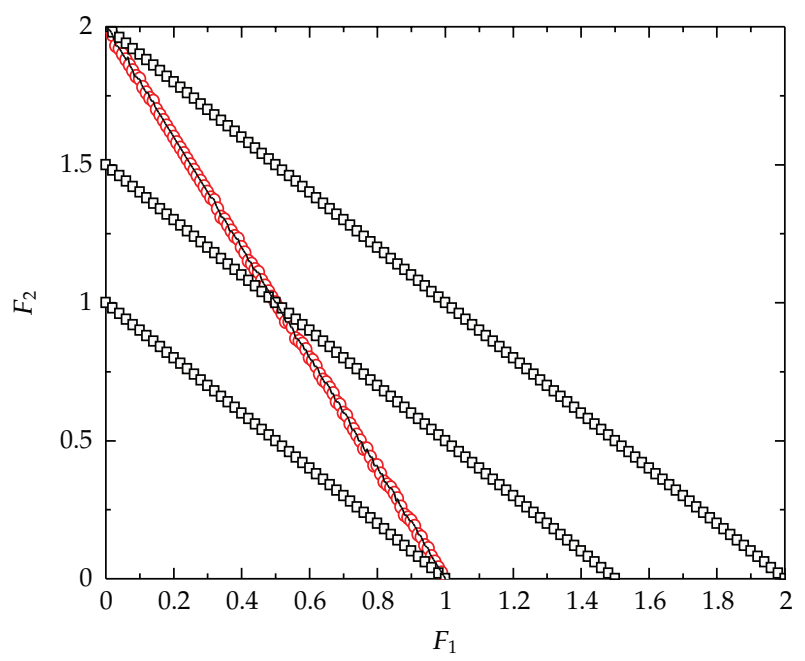

- The obtained upper level Pareto optimal front

- The theoretical Pareto optimal front

․ The theoretical lower level Pareto optimal front when $y_{1}=1$

- The theoretical lower level Pareto optimal front when $y_{1}=1.5$

ㅁ The theoretical lower level Pareto optimal front when $y_{1}=2$

Figure 8: The obtained Pareto front of Example 4.6.

$y_{1}=1.5$ and $y_{1}=2$. It can be seen that only one Pareto optimal point from each participating lower level problem qualifies to be on the upper level Pareto optimal front.

\subsubsection{The BLMPPs with Unknown Theoretical Pareto Optimal Fronts}

Example 4.7. Example 4.7 is taken from [52], in which the theoretical Pareto optimal front is not given. Here $x \in R^{2}, y \in R^{3}$. In this example, the population size and iteration times are set as follows: $N_{u}=100, T_{u}=50, N_{l}=20, T_{l}=10$, and $T=40$ :

$$
\begin{array}{ll}
\max _{x} & F(x, y)=\left(x_{1}+9 x_{2}+10 y_{1}+y_{2}+3 x_{3}, 9 x_{1}+2 x_{2}+2 y_{1}+7 y_{2}+4 x_{3}\right) \\
\text { s.t. } & G_{1}(x, y)=3 x_{1}+9 x_{2}+9 y_{1}+5 y_{2}+3 y_{3} \leq 1039 \\
& G_{2}(x, y)=-4 x_{1}-x_{2}+3 y_{1}-3 y_{2}+2 y_{3} \leq 94 \\
\min _{y} & f(x, y)=\left(4 x_{1}+6 x_{2}+7 y_{1}+4 y_{2}+8 y_{3}, 6 x_{1}+4 x_{2}+8 y_{1}+7 y_{2}+4 y_{3}\right) \\
\text { s.t. } & g_{1}(x, y)=3 x_{1}-9 x_{2}-9 y_{1}-4 y_{2} \leq 61 \\
& g_{2}(x, y)=5 x_{1}+9 x_{2}+10 y_{1}-y_{2}-2 y_{3} \leq 924 \\
& g_{3}(x, y)=3 x_{1}-3 x_{2}+y_{2}+5 y_{3} \leq 420 \\
& x_{1}, x_{2}, y_{1}, y_{2}, y_{3} \geq 0 .
\end{array}
$$


Example 4.8. Example 4.8 is taken from [23]. Here $x \in R^{1}, y \in R^{2}$. In this example, the population size and iteration times are set as follows: $N_{u}=800, T_{u}=50, N_{l}=40, T_{l}=20$, and $T=40$ :

$$
\begin{aligned}
\min _{x} F(x, y) & =\left(x+y_{1}^{2}+y_{2}^{2}+\sin ^{2}\left(x_{1}+y\right), \cos \left(y_{2}\right)(0.1+y)\left(\exp \left(-\frac{y_{1}}{0.1+y_{2}}\right)\right)\right) \\
\text { s.t. } \quad G_{1}(x, y)= & (x-5)^{2}-\left(y_{1}-0.5\right)^{2}-\left(y_{2}-5\right)^{2} \leq 16 \\
\min _{y} f(x, y)= & \left(\frac{\left(y_{1}-2\right)^{2}+\left(y_{2}-2\right)^{2}}{4}+\frac{x y_{2}+(5-x)^{2}}{16}\right. \\
& \left.+\sin \left(\frac{y_{2}}{10}\right), \frac{y_{1}^{2}+\left(y_{2}-6\right)^{2}-2 x y_{1}-(5-x)^{2}}{80}\right)
\end{aligned}
$$

s.t. $\quad g_{1}(x, y)=y_{1}^{2}-y_{2} \leq 0$

$$
\begin{aligned}
& g_{2}(x, y)=5 y_{1}^{2}+y_{2}-10-\leq 0 \\
& g_{3}(x, y)=y_{2}-5-\frac{x}{6} \leq 0 \\
& 0 \leq x \leq 10, \quad 0 \leq y_{1}, \quad y_{2} \leq 10 .
\end{aligned}
$$

Figure 9 shows the obtained Pareto optimal front of Example 4.7 by the proposed algorithm. Note that, Zhang et al. [52] only obtained a single optimal solution $x=(146.2955,28.9394)$ and $y=(0,67.9318,0)$ which lies on the maximum of the $F_{2}$ using weighted sum method. In contrast, a set of Pareto optimal solutions are obtained by the proposed algorithm. However, the fact that the single optimal solution in [49] is included in the obtained Pareto optimal solutions illustrates the feasibility of proposed algorithm. Figure 10 shows the final archive solutions of the Example 4.8 by the proposed algorithm. For this problem, the exact Pareto optimal front is not known, but the obtained Pareto optimal front by the proposed algorithm is similar to that reported in the previous study [23].

\section{Conclusion}

In this paper, an EQPSO is presented, in which an elite strategy is exerted for global best particle to prevent the swarm from clustering, enabling the particle to escape the local optima. The EQPSO algorithm is employed for solving bilevel multiobjective programming problem (BLMPP) for the first time. In this study, some numerical examples are used to explore the feasibility and efficiency of the proposed algorithm. The experimental results indicate that the obtained Pareto front by the proposed algorithm is very close to the theoretical Pareto optimal front, and the solutions are also distributed uniformly on entire range of the theoretical Pareto optimal front. The proposed algorithm is simple and easy to implement, which provides another appealing method for further study on BLMPP. 


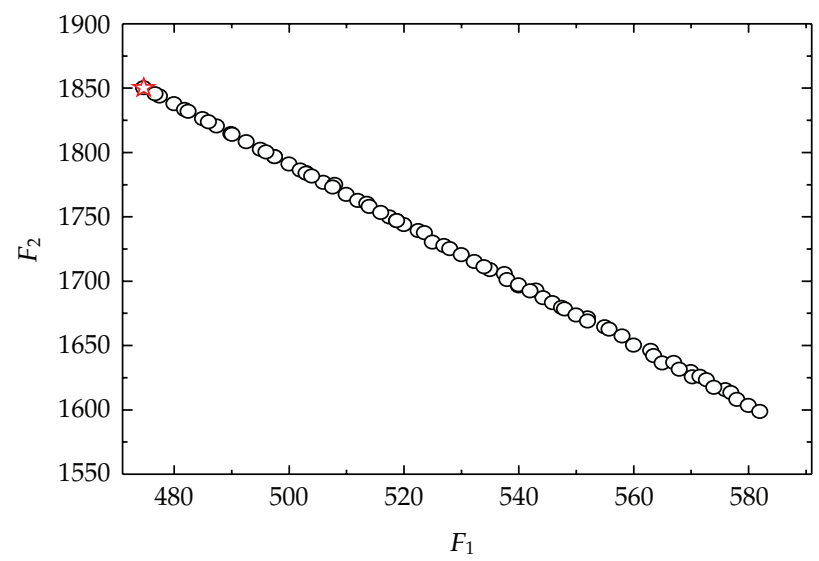

○ The obtained Pareto optimal front by the proposed algorithm

The single optimal solution in [37]

Figure 9: The obtained front of Example 4.7.

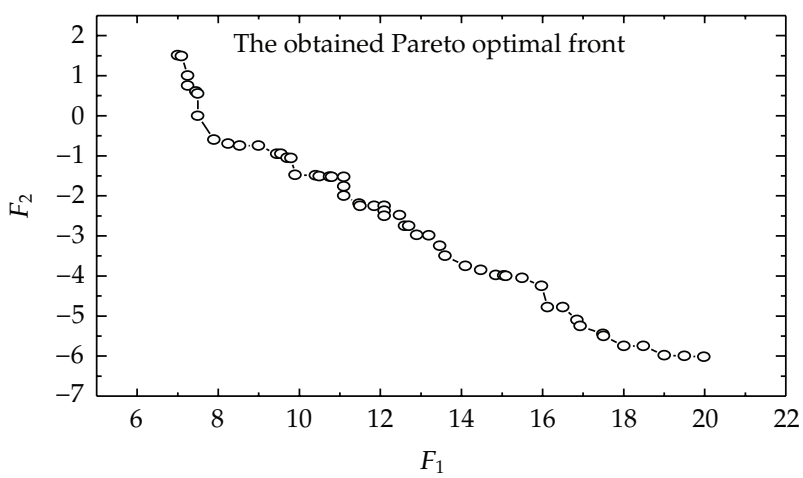

Figure 10: The obtained front of Example 4.8.

\section{Acknowledgments}

The authors are indebted to the referees and the associate editor for their insightful and pertinent comments. This work is supported by the National Science Foundation of China (71171150, 71171151, 50979073, 61273179, 11201039, 20101304), the Academic Award for Excellent Ph.D. Candidates Funded by Wuhan University and the Fundamental Research Fund for the Central Universities (no. 201120102020004), and the Ph.D. short-time mobility program by Wuhan University.

\section{References}

[1] B. Colson, P. Marcotte, and G. Savard, "An overview of bilevel optimization," Annals of Operations Research, vol. 153, pp. 235-256, 2007.

[2] L. N. Vicente and P. H. Calamai, "Bilevel and multilevel programming: a bibliography review," Journal of Global Optimization, vol. 5, no. 3, pp. 291-306, 1994.

[3] J. F. Bard, Practical Bilevel Optimization: Algorithms and Applications, Kluwer Academic Publishers, Dordrecht, The Netherlands, 1998. 
[4] S. Dempe, Foundations of Bilevel Programming, vol. 61 of Nonconvex Optimization and Its Applications, Kluwer Academic Publishers, Dordrecht, The Netherlands, 2002.

[5] S. Dempe, "Annotated bibliography on bilevel programming and mathematical programs with equilibrium constraints," Optimization, vol. 52, no. 3, pp. 333-359, 2003.

[6] Z.-Q. Luo, J.-S. Pang, and D. Ralph, Mathematical Programs with Equilibrium Constraints, Cambridge University Press, Cambridge, UK, 1996.

[7] K. Shimizu, Y. Ishizuka, and J. F. Bard, Non-differentiable and Two-Level Mathematical Programming, Kluwer Academic Publishers, Dordrecht, The Netherlands, 1997.

[8] B. Colson, P. Marcotte, and G. Savard, "Bilevel programming: a survey," A Quarterly Journal of Operations Research, vol. 3, no. 2, pp. 87-107, 2005.

[9] G. M. Wang, Z. P. Wan, and X. J. Wang, "Bibliography on bilevel programming," Advances in Mathematics, vol. 36, no. 5, pp. 513-529, 2007 (Chinese).

[10] L. N. Vicente and P. H. Calamai, "Bilevel and multilevel programming: a bibliography review," Journal of Global Optimization, vol. 5, no. 3, pp. 291-306, 1994.

[11] J. W. Chen, Y. J. Cho, J. K. Kim, and J. Li, "Multiobjective optimization problems with modified objective functions and cone constraints and applications," Journal of Global Optimization, vol. 49, no. 1, pp. 137-147, 2011.

[12] J. W. Chen, Z. Wan, and Y. J. Cho, "Nonsmooth multiobjective optimization problems and weak vector quasi-variational inequalities," Computational and Applied Mathematics. In press.

[13] R. Mathieu, L. Pittard, and G. Anandalingam, "Genetic algorithm based approach to bi-level linear programming," RAIRO Recherche Opérationnelle, vol. 28, no. 1, pp. 1-21, 1994.

[14] V. Oduguwa and R. Roy, "Bi-level optimization using genetic algorithm," in Proceedings of the IEEE International Conference on Artificial Intelligence Systems, pp. 322-327, 2002.

[15] Y. Wang, Y. C. Jiao, and H. Li, "An evolutionary algorithm for solving nonlinear bilevel programming based on a new constraint-handling scheme," IEEE Transactions on Systems, Man and Cybernetics C, vol. 35, no. 2, pp. 221-232, 2005.

[16] Y. Yin, "Genetic-algorithms-based approach for bilevel programming models," Journal of Transportation Engineering, vol. 126, no. 2, pp. 115-119, 2000.

[17] X. Shi and H. Xia, "Interactive bilevel multi-objective decision making," Journal of the Operational Research Society, vol. 48, no. 9, pp. 943-949, 1997.

[18] X. Shi and H. Xia, "Model and interactive algorithm of bi-level multi-objective decision-making with multiple interconnected decision makers," Journal of Multi-Criteria Decision Analysis, vol. 10, pp. 27-34, 2001.

[19] M. A. Abo-Sinna and I. A. Baky, "Interactive balance space approach for solving multi-level multiobjective programming problems," Information Sciences, vol. 177, no. 16, pp. 3397-3410, 2007.

[20] I. Nishizaki and M. Sakawa, "Stackelberg solutions to multiobjective two-level linear programming problems," Journal of Optimization Theory and Applications, vol. 103, no. 1, pp. 161-182, 1999.

[21] Y. Zheng, Z. Wan, and G. Wang, "A fuzzy interactive method for a class of bilevel multiobjective programming problem," Expert Systems with Applications, vol. 38, no. 8, pp. 10384-10388, 2011.

[22] G. Eichfelder, "Solving nonlinear multi-objective bi-level optimization problems with coupled upper level constraints," Tech. Rep. 320, University of Erlangen-Nuremberg, Erlangen, Germany, 2007, Preprint-Series of the Institute of Applied Mathematics.

[23] G. Eichfelder, "Multiobjective bilevel optimization," Mathematical Programming, vol. 123, no. 2, pp. 419-449, 2010.

[24] K. Deb and A. Sinha, "Constructing test problems for bilevel evolutionary multi-objective optimization," in Proceedings of the IEEE Congress on Evolutionary Computation (CEC '09), pp. 1153 1160, May 2009.

[25] K. Deb and A. Sinha, "Solving bilevel multi-objective optimization problems using evolutionary algorithms," Evolutionary Multi-Criterion Optimization, vol. 5467, pp. 110-124, 2009.

[26] K. Deb and A. Sinha, "An evolutionary approach for bilevel multi-objective problems," Communications in Computer and Information Science, vol. 35, pp. 17-24, 2009.

[27] A. Sinha and K. Deb, "Towards understanding evolutionary bilevel multiobjective optimization algorithm," Tech. Rep. Kangal Report No. 2008006, Department of Mechanical Engineering, Indian Institute of Technology Kanpur, Kanpur, India, 2008, http:/ /www.iitk.ac.in/kangal/reports.shtml.

[28] K. Deb and A. Sinha, "An efficient and accurate solution methodology for bilevel multi-objective programming problems using a hybrid evolutionary local-search algorithm," Tech. Rep. Kangal Report No. 2009001, 2009, http:/ / www.iitk.ac.in/kangal/reports.shtml. 
[29] A. Sinha, "Bilevel multi-objective optimization problem solving using progressively interactive EMO," in Proceeding of the 6th International Conference on Evolutionary Multi-Criterion Optimization, pp. 269-284, 2011.

[30] J. Kennedy, R. Eberhart, and Y. Shi, Swarm intelligence, Morgan Kaufmann, San Francisco, Calif, USA, 2001.

[31] X. Li, P. Tian, and X. Min, "A hierarchical particle swarm optimization for solving bilevel programming problems," in Proceedings of the 8th International Conference on Artificial Intelligence and Soft Computing (ICAISC'06), pp. 1169-1178, 2006.

[32] R. J. Kuo and C. C. Huang, "Application of particle swarm optimization algorithm for solving bi-level linear programming problem," Computers \& Mathematics with Applications, vol. 58, no. 4, pp. 678-685, 2009.

[33] Y. Gao, G. Zhang, J. Lu, and H.-M. Wee, "Particle swarm optimization for bi-level pricing problems in supply chains," Journal of Global Optimization, vol. 51, no. 2, pp. 245-254, 2011.

[34] F. V. D. Bergh, An analysis of particle swarm optimizers [Ph.D. thesis], Department of Computer Science, University of Pretoria, Pretoria, South Africa, 2001.

[35] J. Sun, B. Feng, and W. Xu, "Particle swarm optimization with particles having quantum behavior," in Proceedings of the 2004 Congress on Evolutionary Computation, pp. 325-331, June 2004.

[36] J. Sun, W. B. Xu, and B. Feng, "A global search strategy of Quantum-behaved Particle Swarm Optimization," in Proceedings of the IEEE Conference on Cybernetics and Intelligent Systems, pp. 111-116, December 2004.

[37] J. Sun, W. B. Xu, and B. Feng, “Adaptive parameter control for quantum-behaved particle swarm optimization on individual level," in Proceedings of the IEEE International Conference on Systems, Man and Cybernetics, pp. 3049-3054, October 2005.

[38] J. Sun, W. Xu, and W. Fang, "Quantum-behaved particle swarm optimization with a hybrid probability distribution," in Proceedings of the 9th Pacific Rim International Conference on Artificial Intelligence, pp. 737-746, August 2006.

[39] W. Fang, J. Sun, Z. P. Xie, and W. B. Xu, "Convergence analysis of quantum-behaved particle swarm optimization algorithm and study on its control parameter," Acta Physica Sinica, vol. 59, no. 6, pp. 3686-3694, 2010.

[40] L. D. S. Coelho, "Gaussian quantum-behaved particle swarm optimization approaches for constrained engineering design problems," Expert Systems with Applications, vol. 37, no. 2, pp. 1676-1683, 2010.

[41] S. N. Omkar, R. Khandelwal, T. V. S. Ananth, G. Narayana Naik, and S. Gopalakrishnan, "Quantum behaved Particle Swarm Optimization (QPSO) for multi-objective design optimization of composite structures," Expert Systems with Applications, vol. 36, no. 8, pp. 11312-11322, 2009.

[42] S. L. Sabat, L. dos Santos Coelho, and A. Abraham, "MESFET DC model parameter extraction using Quantum Particle Swarm Optimization," Microelectronics Reliability, vol. 49, no. 6, pp. 660-666, 2009.

[43] H. Shayeghi, H. A. Shayanfar, S. Jalilzadeh, and A. Safari, "Tuning of damping controller for UPFC using quantum particle swarm optimizer," Energy Conversion and Management, vol. 51, no. 11, pp. 2299-2306, 2010.

[44] C. Sun and S. Lu, "Short-term combined economic emission hydrothermal scheduling using improved quantum-behaved particle swarm optimization," Expert Systems with Applications, vol. 37, no. 6, pp. 4232-4241, 2010.

[45] Z. Zhisheng, "Quantum-behaved particle swarm optimization algorithm for economic load dispatch of power system," Expert Systems with Applications, vol. 37, no. 2, pp. 1800-1803, 2010.

[46] L. D. S. Coelho, "A quantum particle swarm optimizer with chaotic mutation operator," Chaos, Solitons E Fractals, vol. 37, no. 5, pp. 1409-1418, 2008.

[47] M. Xi, J. Sun, and W. B. Xu, “An improved quantum-behaved particle swarm optimization algorithm with weighted mean best position," Applied Mathematics and Computation, vol. 205, no. 2, pp. 751-759, 2008.

[48] Z. Huang, Y. J. Wang, C. J. Yang, and C. Z. Wu, “A new improved quantum-behaved particle swarm optimization model," in Proceedings of the 4th IEEE Conference on Industrial Electronics and Applications (ICIEA '09), pp. 1560-1564, May 2009.

[49] K. Deb, S. Agarwal, A. Pratap, and T. Meyarivan, "A fast and elitist multiobjective genetic algorithm: NSGA-II," IEEE Transactions on Evolutionary Computation, vol. 6, no. 2, pp. 182-197, 2002.

[50] K. Deb, "Multi-objective optimization using evolutionary algorithms," IEEE Transactions on Evolutionary Computation, vol. 6, pp. 182-197, 2002. 
[51] K. Deb and A. Sinha, "Solving bilevel multi-objective optimization problems using evolutionary algorithms," KanGAL Report, 2008.

[52] G. Zhang, J. Lu, and T. Dillon, "Decentralized multi-objective bilevel decision making with fuzzy demands," Knowledge-Based Systems, vol. 20, no. 5, pp. 495-507, 2007. 


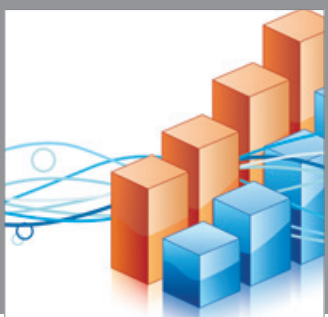

Advances in

Operations Research

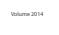

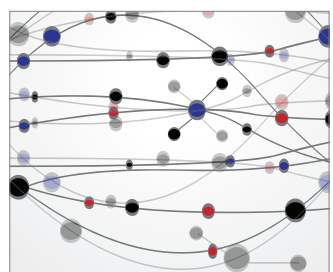

\section{The Scientific} World Journal
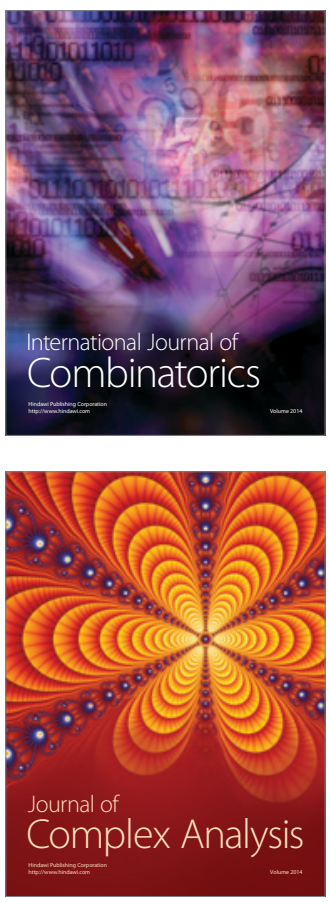

International Journal of

Mathematics and

Mathematical

Sciences
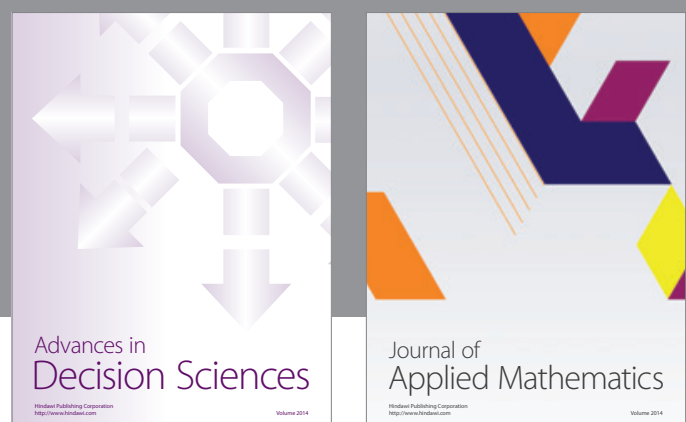

Journal of

Applied Mathematics
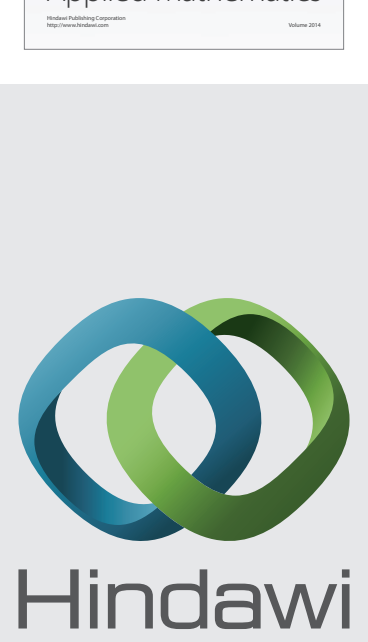

Submit your manuscripts at http://www.hindawi.com
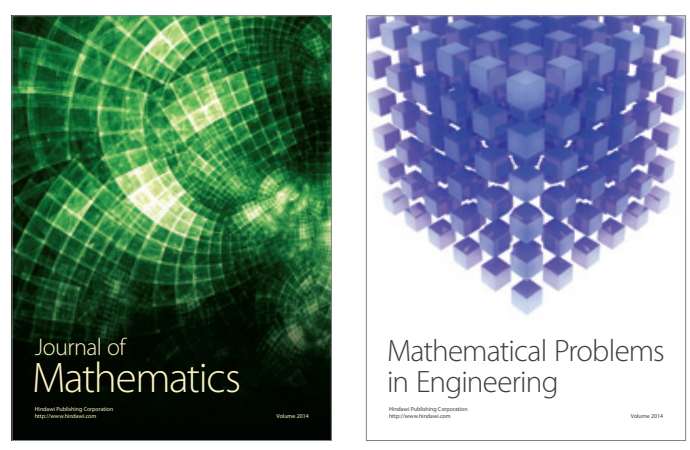

Mathematical Problems in Engineering
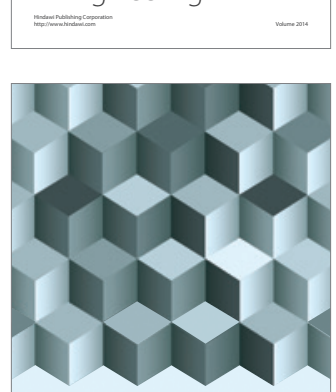

Journal of

Function Spaces
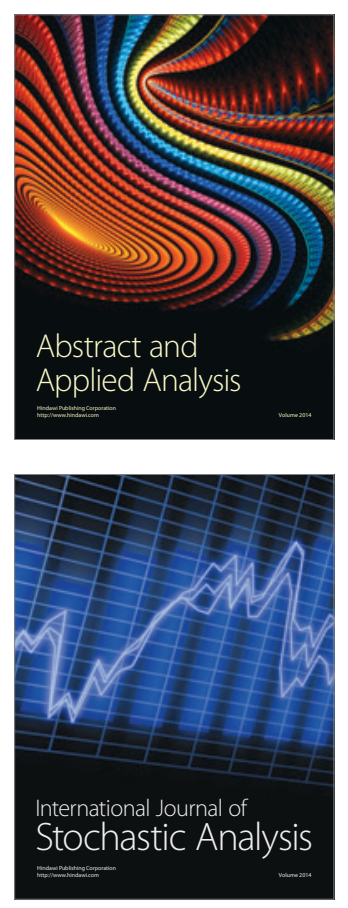

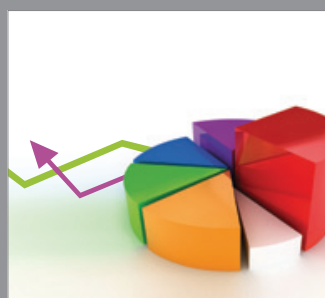

ournal of

Probability and Statistics

Promensencen
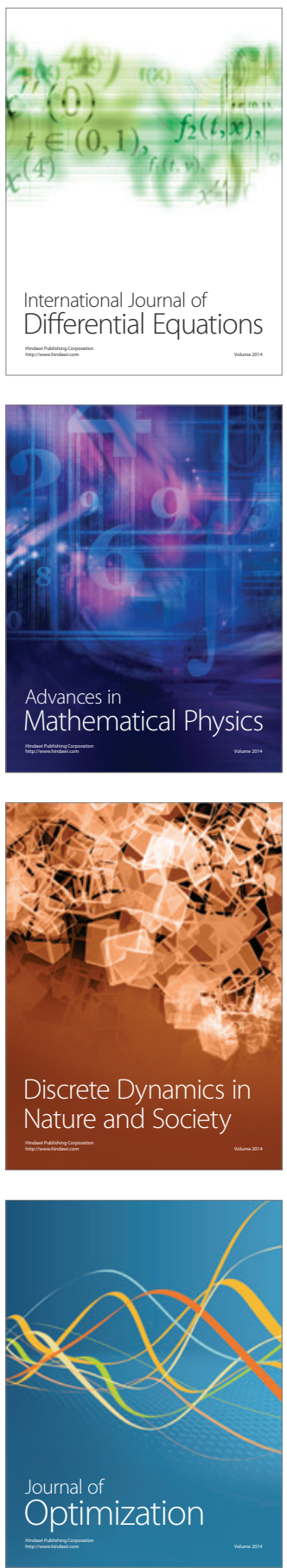